\title{
Enhancement of SAR images using fuzzy shrinkage technique in curvelet domain
}

\author{
SHIVAKUMARA SWAMY PURANIK MATH* and VANI KALIYAPERUMAL \\ Department of Information Science and Technology, Anna University, Chennai 600025, India \\ e-mail: pmsk.swamy68@gmail.com; vani@annauniv.edu
}

MS received 9 May 2015; revised 26 December 2015; accepted 17 January 2017; published 3 August 2017

\begin{abstract}
The synthetic aperture radar (SAR) images are mainly affected by speckle noise. Speckle degrades the features in the image and reduces the ability of a human observer to resolve fine detail, hence despeckling is very much required for SAR images. This paper presents speckle noise reduction in SAR images using a combination of curvelet and fuzzy logic technique to restore speckle-affected images. This method overcomes the limitation of discontinuity in hard threshold and permanent deviation in soft threshold. First, it decomposes noise image into different frequency scales using curvelet transform, and then applies the fuzzy shrinking technique to high-frequency coefficients to restore noise-contaminated coefficients. The proposed method does not use threshold approach only by proper selection of shrinking parameter the speckle in SAR image is suppressed. The experiment is carried out on different resolutions of RISAT-1 SAR images, and results are compared with the existing filtering algorithms in terms of noise mean variance (NMV), mean square difference (MSD), equal number of looks (ENL), noise standard deviation (NSD) and speckle suppression index (SSI). A comparison of the results shows that the proposed technique suppresses noise significantly, preserves the details of the image and improves the visual quality of the image.
\end{abstract}

Keywords. Fuzzy logic; curvelet; speckle; wavelet; SAR image.

\section{Introduction}

Synthetic aperture radar (SAR) system uses active sensors. The active sensors used in SAR radiate electromagnetic waves in the microwave range. Radiations from the SAR system travel in phase and interact with target area. Once it interacts with a target area, it scatters and the wave does not maintain the phase even though they are coherent in frequency. This is due to the differences in distance, the wave travel back from different targets, single versus multiple bounce scattering due to variance in the surface roughness. The waves go out of phase when the synthesised antenna is moved; these out-of-phase waves interfere constructively or destructively to produce stronger or weaker signals at the antenna of the SAR system [1]. When the image is constructed from the backscattered pulses by the SAR system, there will be variations of intensity from pixel to pixel, which forms a salt-andpepper granular pattern during the construction image called speckle noise [2].

The speckle noise has a standard deviation linearly related to the mean often modeled as a multiplicative process; it means that the higher the signal strength, then the higher the amount of noise. This indicates more speckle noise is commonly present

*For correspondence near brighter pixel areas [1]. The presence of speckle in the image reduces the ability of a human observer to resolve fine details, and leads to misinterpretation of objects [2]. Therefore, speckle reduction is an important preprocessing step in SAR image processing. In order to remove speckles in SAR image, many denoising techniques are used. The denoising methods include spatial domain and transform domain filters [3]. Some of the major filters are in spatial domain: Lee [4], Frost et al [5], Kuan et al [6], Gamma maximum a posteriori (MAP) [7] and median [8]. The spatial domain filters will remove speckle to a certain extent; but at the same time, they may not preserve the structure of the image [9]. Most transform domain filters implemented with the help of the wavelet transform. Donoho and Johnstone [10] proposed the thresholding approach in the wavelet domain. The hard and soft threshold methods have been widely used in practice to remove speckle noise [11], but they have potential weaknesses. The hard threshold function is discontinuous in the whole wavelet domain or in the curvelet domain and there are interrupted points at threshold. As a result, the image constructed by this method contains residue of noise. In soft thresholding function, a constant deviation exists between the estimated and the original coefficients when the coefficient is greater than the threshold. As a result, an image edge is blurred and the quality of the reconstructed image is reduced [12]. The speckle noise is reduced by using Wavelet Denoising and Markov Random Field Modeling [13], even 
though this algorithm is efficient in suppressing speckle noise but cost estimation of hyper-parameters will be high. The despeckling based on Bayesian estimation and fuzzy shrinkage in wavelet domain is applied to remove speckle in SAR image [14]. Since it involves stationary wavelet transform and expectation maximisation algorithm, computation will be highly complex. A statistical approach for denoising of SAR images is used to suppress speckle noise in wavelet domain [15]; this method suffers from shift-variance resulting from decimated wavelet and computational concerns of the complex model. A stationary wavelet-domain wiener filter for correlated speckle was proposed to despeckle [16], this approach assumes homogeneous statistics and smoothes the homogeneous region. Homogeneous assumption limits the filtering performance, and it does not work well in the heterogeneous areas. The transformed filters discussed adopt wavelet transformation. Since the wavelet is lacking in representing edges significantly along curves, this may limit the representation of the boundaries of the features. These limitations of wavelet are overcome by the curvelet transform [17]. The curvelet transform will represent edges and curves in images well with fewer coefficients. By considering the limitations of hard threshold, soft threshold and wavelet transform, a fuzzy logic shrinking is proposed in the curvelet domain so that it overcomes the limitations of threshold (hard and soft) and wavelet transform. Fuzzy logicbased shrinking technique used in this work will maintain continuity instead of discontinuity as in hard threshold. As coefficient values are increasing, the deviation between the estimated and original coefficient will be reduced to overcome the limitation of soft threshold. The curvelet transform that is used in this work is fast discrete curvelet transformation via wrapping technique. This transformation overcomes the shift invariance limitation of wavelet transform. This method suppresses speckle noise in SAR images by combining curvelet and fuzzy logic. The experimental results of the proposed method are compared with wavelet fuzzy shrinkage [18], curvelet denoising [19], curvelet cycle spin technique [20], and curvelet improved soft threshold technique [21].

\section{Noise model}

The speckle noise has a standard deviation that is linearly related to the mean and is often modeled as a multiplicative process. This indicates that the higher the signal strength, the higher is the noise. As a result, more speckle noise is commonly present near brighter pixel areas. The curvelet denoising is based on additive Gaussian noise. Therefore, the speckle noise must be turned into additive noise to perform denoising [22]. The speckle noise is converted to the additive noise with the help of homomorphic transform by applying the logarithmic operator.

The model of the noisy image is given as

$$
I_{n}=I_{s} \eta
$$

The multiple nature of speckle noise can be converted into additive by applying the logarithm of both sides of Eq. (1)

$$
\log \left(I_{n}\right)=\log \left(I_{s}\right)+\log (\eta)
$$

where $I_{n}$ is the noisy image, $I_{s}$ is the unknown original image and $\eta$ is the noise.

\section{Methodology}

The speckle removal process is shown in figure 1. The speckle noise is multiplicative, so a denoising method cannot be applied directly. Convert multiplicative noise into additive by applying the logarithm to a noisy image using Eq. (2). Next, curvelet transform transforms the image into coarse, detail and fine scale. Since the coarse scale contains lowfrequency components (image information), the denoising process is applied only on detail and fine scales.

For each scale, coefficient membership is assigned using Eq. (11). Once the membership is assigned, the next process is related to suppressing the noise. The important step in fuzzy denoising lies in the modification of membership values. The membership values are modified by appropriate fuzzy techniques, such as fuzzy clustering, fuzzy rule-based approach, and fuzzy integration approach. In the proposed work, the fuzzy membership is modified using Eq. (12). After the membership value is modified defuzzification process is applied with the help of Eq. (13). Denoised coefficients are obtained as outputs of the defuzzifier. Inverse curvelet transform is applied to denoised coefficients, and exponential operation is performed to obtain the denoised image.

\section{Curvelet transform and fuzzy logic}

The most denoising filters in the frequency domain will use the wavelet and Fourier transform. But these transforms suffer from shift, rotation sensitivity and poor

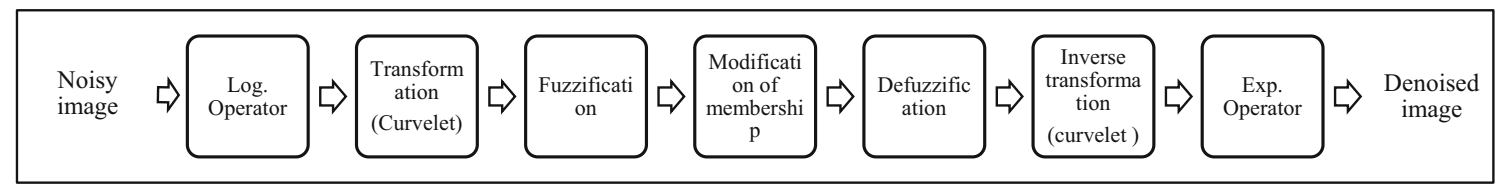

Figure 1. Block diagram of denoising. 
directionality. The curvelet transform is a multi-scale geometric transform. It is developed to overcome the limitations of wavelet transform and Fourier transforms [20]. The curvelet transform was designed to represent edges and other singularities along curves much more efficiently than the wavelet transform [17, 23]. The wavelet approximately requires $1 / N$ wavelets to represent an edge, whereas curvelet transform requires only $1 / \sqrt{ } N$ curvelets [19]. Curvelet transform is based on anisotropic scaling, and wavelet is based on isotropic scaling. The elements of curvelet will follow the relation width $\approx$ length $^{2}$ [19]. The curvelet transform is a multi-scale pyramid with many directions and positions of each scale and needle-shaped elements at fine scales [17]. It decomposes the signal into several subbands at each scale with different orientations and positions in the frequency domain [17]. Further discussion of curvelet transforms in detail follows.

In Cartesian coordinates, taking $f\left[t_{1}, t_{2}\right], 0 \leq t_{1}$, and $t_{2}<$ $n$ as input, the digital curvelet transform $c^{D}(j, k, l)$ is obtained as

$$
c^{D}(j, k, l)=\sum_{0 \leq t_{1, t_{2}}<n} f\left[t_{1}, t_{2}\right] \overline{\varphi_{j, l, k}^{D}\left[t_{1}, t_{2}\right]}
$$

where $\overline{\varphi_{j, l, k}^{D}\left[t_{1}, t_{2}\right]}$ is the digital curvelet waveform.

\subsection{Curvelet transform via wrapping technique}

The curvelet transform in this work is a fast discrete curvelet transformed via the wrapping technique. This new discrete curvelet transforms is simpler, quicker and less redundant compared to the first-generation version [17].

The steps for fast discrete curvelet transform via the wrapping technique are as follows:

1. For a given two-dimensional function, which is defined in Cartesian coordinate, a 2D FFT (two dimensional fast fourier transform) is applied to obtain $\hat{f}\left[n_{1}, n_{2}\right]$, $-n / 2 \leq n_{1}, n_{2} \leq n / 2$.

2. For each scale $j$ and angle $l$ in the frequency domain, $\hat{f}\left[n_{1}, n_{2}\right]$ is re-sampled to obtain $\hat{f}\left(n_{1}, n_{2}\right)$, $\hat{f}\left(n_{1}, n_{2}-n_{1} \tan \theta_{l}\right)\left(n_{1}, n_{2}\right) \in P_{j}$.

3. The sampled value is multiplied by the window function $\tilde{U}_{j}$ to obtain $\hat{f}\left(n_{1}, n_{2}\right)=\hat{f}\left[n_{1}, n_{2}-n_{1} \tan \theta_{l}\right] \tilde{U}_{j}\left[n_{1}, n_{2}\right]$.

4. $\hat{f}\left[n_{1}, n_{2}\right]$ is wrapped around the origin to obtain $\hat{f}\left[n_{1}, n_{2}\right]=W\left(\tilde{U}_{j, l} \hat{f}\right)\left[n_{1}, n_{2}\right]$, here the ranges of $n_{1}$ and $n_{2}$ are now $0 \leq n_{1}<\mathrm{L} 1, j$ and $0 \leq n_{2}<\mathrm{L} 2, j$, and range of $\theta$ is $-\frac{\pi}{4}<\theta<\frac{\pi}{4}$.

5. The inverse 2D FFT is applied to each $f_{j . l}$, after obtaining the discrete curvelet transform coefficients, $c^{D}(j, k, l)$.

The curvelet transform decomposes the image into different number of scale layers with different orientation at each scale. The scale height depends on the size of the image. The scale layers are coarse, detail and fine layers based on the frequency from low to high. The coarse layer contains low-frequency coefficients. The detail layer is an intermediate one, and it contains intermediate-frequency coefficients. The fine layer is the outer layer (high-frequency layer), and it provides edge and detail information of the original image [24]. The low-frequency coefficients are less contaminated by noise, but high-frequency coefficients are highly contaminated by noise. The low-frequency coefficients carry image information, therefore only highfrequency coefficients are processed to remove the noise. The noise in case of curvelet transform is present in coefficients having lower values and coefficients having higher value will indicate information. Therefore, in order to remove noise, proper function needs to be selected to suppress lower-value coefficients.

\subsection{Fuzzy set theory}

The fuzzy set theory has been adopted by more and more recent intelligent systems due to its simplicity and similarity to human reasoning [25]. Fuzzy set theory is the improvement of conventional set theory. It deals with only true and false, that is, a member either belongs to or not belongs to set. It does not deal with partial truth or false. The fuzzy set theory will handle the concept of partial truth and false. The complete truth value is 1 and complete false value is 0 and partial truth and false lies between 0 and 1 .

The generalised fuzzy set in universe $U$ is defined as

$$
A=\int \mu_{A} / x
$$

where $x \in \mu$.

$\mu_{A}(x)$ will be in the range of $[-1,1]$ for a generalised membership function.

Generalised fuzzy set is when U contains finite elements, it is defined as

$$
\begin{gathered}
A=\frac{\mu_{A}\left(x_{1}\right)}{x_{1}}+\frac{\mu_{A}\left(x_{2}\right)}{x_{2}}+\cdots+\frac{\mu_{A}\left(x_{n}\right)}{x_{n}}, \\
A=\bigcup_{i=1}^{n} \frac{\mu_{A}\left(x_{i}\right)}{x_{i}}
\end{gathered}
$$

$\mu_{A}\left(x_{i}\right)$ is the generalised membership function $x_{i}$ in $\mathrm{U}$.

In normal fuzzy set the membership lies between $[0,1]$, but in generalised fuzzy set membership lies between $[-1,1]$.

\section{Threshold functions}

The basic threshold functions used in transform domain are hard and soft thresholds. These thresholds are discussed in the subsections. 


\subsection{Hard threshold}

The hard threshold compares the absolute value of each coefficient with the threshold, if the coefficient is less than the threshold, then the coefficient value will be shrunk to zero otherwise coefficient value is retained.

$$
f(x)= \begin{cases}x, & |x|>t h \\ 0, & |x| \leq t h\end{cases}
$$

where $x$ is the curvelet coefficient, and th is the threshold and it is given in Eq. (9).

\subsection{Soft threshold}

The soft threshold compares the absolute value of each coefficient with threshold; if the absolute value of the coefficient is greater than the threshold, then shrinks coefficient in absolute value, otherwise shrinks to zero.

$$
f(x)=\left\{\begin{array}{cc}
\operatorname{sign}(x) \operatorname{Max}(0,|x|-t h), & |x|>t h \\
0, & |x| \leq t h
\end{array}\right.
$$

where $t h$ is the threshold and it is given by

$$
t h=\sigma_{w} k_{1} \sigma_{n}
$$

$\sigma_{n}$ is the standard deviation of noise and it is obtained by using the robust median estimator from the diagonal subband coefficients of wavelet [10]; it is given by

$$
\sigma_{n}=\frac{\text { median }\left|w_{i, j}\right|}{0.6745}
$$

$w_{i, j}$ is the finest or diagonal sub-band coefficients of wavelet transform.

To estimate the noise variance $\sigma_{w}^{2}$ of each curvelet index, the Monte Carlo simulation method is used [19]. Few standard white noise images, with zero mean and variance one, i.e., $N(0,1)$, were discrete curvelet transformed and the variance, $\sigma_{w}^{2}$ is estimated. $k_{1}$ is a scaledependent value, $\left(k_{1}=4\right)$ for the fine scale, $\left(k_{1}=3\right)$ for detail scales [19].

\section{Proposed fuzzy shrinkage function}

The curvelet coefficients of each scale are assigned with the fuzzy membership using Eq. (11) based on the magnitude. The membership will be allocated between $[-1,1]$, which is a generalised fuzzy interval. The higher the magnitude, the higher is the membership value, and it indicates a corresponding coefficient belongs to signal, lesser magnitude will have lower membership value, and the corresponding coefficient belongs to noise. The fuzzy logic process has three main stages: (i) fuzzification (converting coefficients into fuzzy members), (ii) modification of membership values, and (iii) defuzzification (converting back to coefficients). The fuzzy logic denoising process is as follows.

The fuzzy membership equation is given by

$$
\mu_{i, j}=\frac{c_{i . j}}{M A X}, \quad M A X=\max \left\{\left|c_{i, j}\right|\right\}
$$

where $c_{i, j}$ is the curvelet coefficient in all scales except first scale, and the absolute value $\mu_{i, j}\left(\left|\mu_{i, j}\right| \in[0,1]\right)$ representing the degree the coefficient belongs to signal.

The fuzzy shrinkage function is defined in Eq. (12); it adjusts fuzzy membership values for all coefficients in high-frequency scales.

$$
\overline{\mu_{i, j}}=\left\{\begin{array}{c}
-\left(-2 \cdot \mu_{i, j}-\mu_{i, j}^{2}\right)^{k},-1 \leq \mu_{i, j}<0 \\
\left(2 \cdot \mu_{i, j}-\mu_{i, j}^{2}\right)^{k}, 0 \leq \mu_{i, j} \leq 1
\end{array}\right.
$$

where $\overline{\mu_{i, j}}$ is the new membership value, $k$ is the shrinking parameter and it is a scalar. It changes the degree of membership and also controls the degree of shrinkage. The value of $k$ is always greater than one. Since the $k$ is power, it controls the membership value. In fuzzy shrinking function if $\left|\mu_{i j}\right|$ value is low, then the modified membership value $\left|\overline{\mu_{i, j}}\right|$ shrinks much nearer to zero value, this is because of $k$. The coefficients which are arriving lower membership are corresponds to noise, hence shrinking lower value membership coefficients will reduce the noise. When membership values are higher the corresponding members are approximated to the same value as input membership value, hence retaining the information. As observed from the graph, as input membership values increase the modified output membership values gradually increase and maintain continuity. At higher values of membership, the output memberships are almost same as input membership. The value of $k$ can be adjusted depending on the different images to make the fuzzy shrinkage function for removing the noise present in the images.

The scalar $k$ is assumed as 4 and threshold $t h$ is equal to 0.2 for plotting the curve. The fuzzy shrinkage curve is as shown in figure 2 and compared with hard and soft

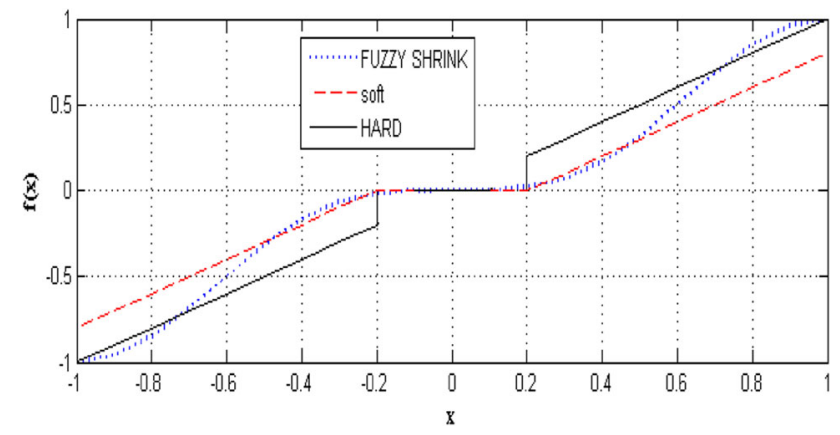

Figure 2. Different thresholding functions. (a) Hard, (b) soft and (c) fuzzy shrinkage. 
threshold function. As observed from the graph in hard threshold, if curvelet coefficient is less than the threshold the coefficient shrinks to zero, but in fuzzy function it approximates nearer to zero. As coefficient values are greater than a threshold, the output coefficients are same as input coefficient, but the abrupt transition takes place at the threshold. This abrupt transition may introduce residue of noise. In case of fuzzy function, the abrupt rise is avoided and increases output value gradually.

In soft threshold, when coefficient value is less than the threshold, it behaves as hard threshold. When coefficient value is greater than the threshold, the output coefficient value is subtracted from the threshold, and avoids abrupt transition as found in hard threshold. Since coefficient values are greater than the threshold, the output coefficients are subtracted from the threshold, hence constant deviation exists. This constant deviation reflects in smoothing of edges. But as observed from fuzzy function, at higher membership values there is no deviation and these values will be almost same as an input membership. Hence it overcomes the constant deviation problem of soft threshold function. Hence, from this discussion, it is found that fuzzy shrinking function will overcome the limitation of hard and soft threshold.

Once the membership values are adjusted, the next process is defuzzification. The defuzzification technique used is maxima technique, and it is given by

$$
\overline{C_{i, j}}=\overline{\mu_{i, j}} \cdot \operatorname{MAX}
$$

where $\overline{C_{i, j}}$ is the noise reduced curvelet coefficient. The maxima technique is convenient for the general fuzzy expert systems. They are computationally efficient and adopt simple multiplication operation.

\section{Results and discussion}

The SAR data used for experimental work is RISAT-1, C-band $(5.35 \mathrm{GHz})$ of medium and fine-resolution images. The images are of Ahmedabad in Gujarat, India. For experimental purpose, the part of the image is taken of size $512 \times 512$, of 8 bit depth; the medium-resolution image is centered on $25^{\circ} 57-72^{\circ} 37$ latitude-longitude, and fine-resolution image centered on $23^{\circ} 44-72^{\circ} 31$ latitude-longitude.

The assessment parameters used by the remote sensing community for measuring despeckling capability of the techniques are noise mean value (NMV), noise standard deviation (NSD), mean square difference (MSD), equal number of looks (ENL) and speckle suppression index (SSI) $[8,9,26,27]$. These parameters will decide the quality of a reconstructed image, the amount of speckle present in the restored image, and performance of the filter technique in suppressing speckle. If NMV and NSD values are less, it indicates less noise present in the denoised image. The higher value of ENL and MSD shows that the quality of the reconstructed image is good, and more speckles being suppressed from uniform and non-uniform regions. The smallest value of SSI indicates minimum speckle noise present in the image. The expressions of assessment parameters are as follows:

$$
\begin{gathered}
N M V=\frac{\sum_{m, n} I_{d}(m, n)}{M \times N} \\
N S D=\sqrt{\frac{\sum_{m, n}\left(I_{d}(m, n)-N M V\right)^{2}}{M \times N}} \\
M S D=\frac{\sum_{m, n}\left(I_{s}(m, n)-I_{d}(m, n)\right)^{2}}{M \times N} \\
S N L=\left[\frac{N M V}{N S D}\right]^{2} \\
\text { ESI }=\frac{\sqrt{\operatorname{var}\left(I_{d}\right)}}{\text { mean }\left(I_{d}\right)} \times \frac{\operatorname{mean}\left(I_{s}\right)}{\sqrt{\operatorname{var}\left(I_{s}\right)}}
\end{gathered}
$$

where $I_{d}(m, n)$ is the despeckled image, $M, N$ are the number of rows and columns of the image respectively, and $I_{s}(m, n)$ is the speckled image.

For the experimental work, the images selected are of size $512 \times 512$ drawn from fine- and medium-resolution image containing heterogeneous region. To find out the performance of the filter the assessment parameters are measured on the whole image, and ENL, SSI measured over uniform regions in an image. On trial and error basis, the value of $k$ is chosen. The scalar $(k=2.5)$ is selected for the experimental purpose.

First, find out the assessment parameters for the whole image. Figure 3 shows the results of denoising by different techniques for a medium-resolution image, and table 1 shows the numerical results of fine- and medium-resolution image for various methods.

The medium-resolution SAR image has NMV of 130.46 and NSD of 38.55 for the proposed approach. These values are small as observed with other approaches. These values indicate less variance and also less deviation in the reconstructed image of the proposed approach; it results in less noise contained in the reconstructed image. The proposed technique has MSD of 1374.39, which is less than the curvelet improved soft technique and is greater than other methods. This indicates that the noise removed by the proposed approach is less than the improved soft threshold approach and greater amount of noise is removed as compared to other approaches. Since the MSD of the curvelet improved soft threshold technique is greater than the proposed method results in smoothening of some regions of the image. The ENL is about 11.45, which is more effective in smoothening the uniform regions and improving the quality of the denoised image. The assessment parameter SSI has 0.653 , which is lower than that of other methods. This shows more amount of speckle removed from the image. 


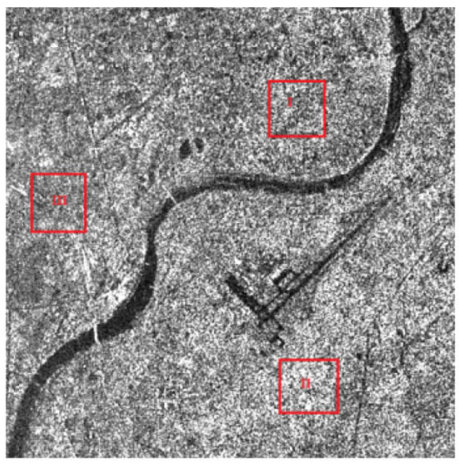

(a)

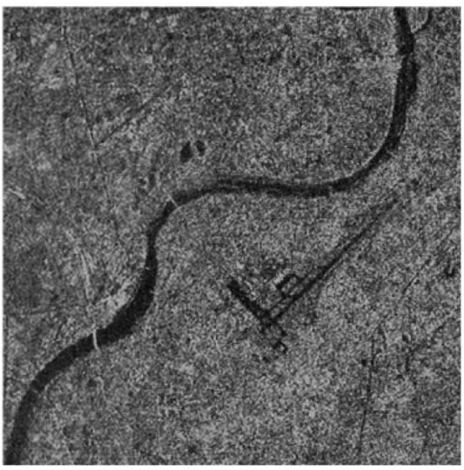

(d)

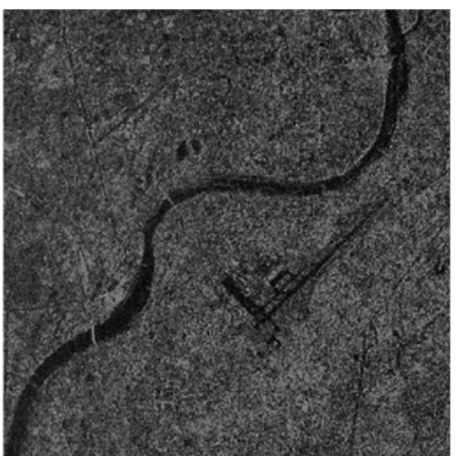

(b)

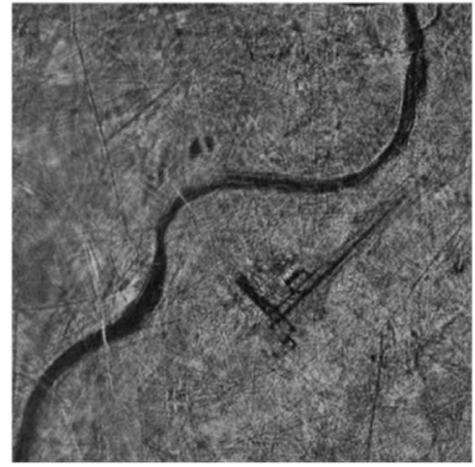

(e)

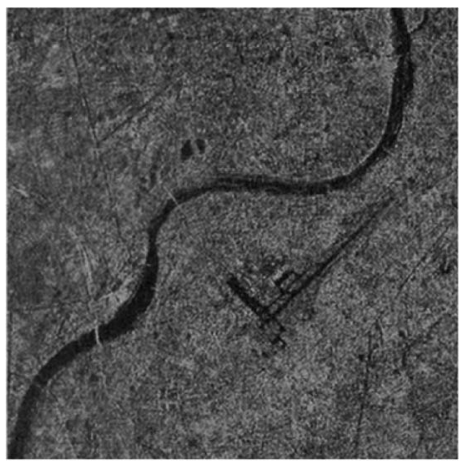

(c)

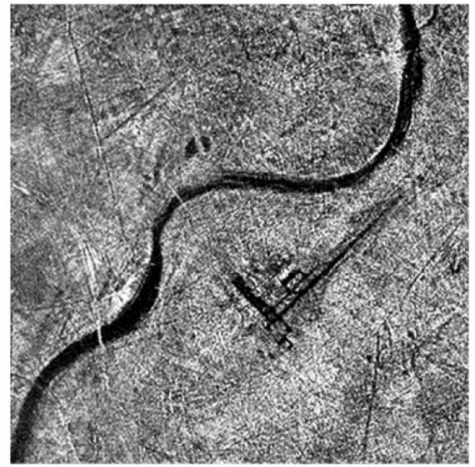

(f)

Figure 3. Denoising results of medium-resolution SAR image. (a) Original image, (b) wavelet fuzzy, (c) curvelet denoising, (d) curvelet cycle spin, (e) curvelet improved soft and (f) curvelet fuzzy.

Table 1. Assessment parameters.

\begin{tabular}{|c|c|c|c|c|c|c|}
\hline Resolution & Techniques & NMV & MSD & ENL & NSD & SSI \\
\hline \multirow{7}{*}{ Medium } & Original image & 140.37 & 0 & 4.89 & 63.47 & 1 \\
\hline & Wavelet fuzzy & 136.19 & 712.67 & 6.14 & 54.92 & 0.891 \\
\hline & Curvelet denoising & 134.24 & 1207.43 & 7.35 & 49.50 & 0.815 \\
\hline & Curvelet cyclespin & 136.95 & 631.87 & 5.76 & 57.05 & 0.921 \\
\hline & Curvelet improved soft & 130.54 & 1723.66 & 11.17 & 39.05 & 0.661 \\
\hline & Curvelet fuzzy & 130.46 & 1374.19 & 11.45 & 38.55 & 0.653 \\
\hline & Original image & 119.24 & 0 & 3.92 & 60.22 & 1 \\
\hline \multirow{5}{*}{ Fine } & Wavelet fuzzy & 117.35 & 363.61 & 4.26 & 56.82 & 0.958 \\
\hline & Curvelet denoising & 118.38 & 142.26 & 4.08 & 58.58 & 0.979 \\
\hline & Curvelet cyclespin & 118.99 & 25.60 & 3.94 & 59.88 & 0.996 \\
\hline & Curvelet improved soft & 117.33 & 249.07 & 4.32 & 56.44 & 0.952 \\
\hline & Curvelet fuzzy & 111.72 & 801.53 & 7.15 & 41.77 & 0.740 \\
\hline
\end{tabular}

To find the effectiveness of the proposed image in a heterogeneous area, a fine-resolution image taken for experimental work which contains urban area and less uniform region as compared to the medium-resolution image. The proposed approach and other techniques are applied to this image and results are tabulated in table 1. The assessment parameter NMV has 111.72 and NSD has 41.77; these values are much lower than those observed with other methods. These values show that the filtered image having less amount of noise. The other parameters MSD and ENL are significantly higher. It indicates that the more the amount of speckle noise removed from the image, the better is the performance of the filter. The SSI has 0.74, which results in more speckle being suppressed from the image as compared with other approaches.

From table 1, it is found that NMV and NSD of the proposed technique are less compared to other methods. This is because the successful speckle-reducing filter will not significantly affect the mean intensity within a homogeneous region.

To measure the effectiveness of the filter in suppressing speckle, the filtering is applied over uniform regions and 
Table 2. ESI and SSI of uniform regions.

\begin{tabular}{|c|c|c|c|c|c|c|c|c|}
\hline \multirow[b]{2}{*}{ Techniques } & \multicolumn{2}{|c|}{ Region I } & \multicolumn{2}{|c|}{ Region II } & \multicolumn{2}{|c|}{ Region III } & \multicolumn{2}{|c|}{ Average } \\
\hline & ENL-I & SSI-I & ENL-II & SSI-II & ENL-III & SSI-III & ENL-avg & SSI-avg \\
\hline Wavelet fuzzy & 8.13 & 0.90 & 7.44 & 0.87 & 11.09 & 0.84 & 8.89 & 0.87 \\
\hline Curvelet denoising & 9.50 & 0.83 & 8.72 & 0.81 & 17.63 & 0.67 & 11.95 & 0.77 \\
\hline Curvelet cyclespin & 8.00 & 0.90 & 6.54 & 0.93 & 12.14 & 0.81 & 8.89 & 0.88 \\
\hline Curvelet improved soft & 14.29 & 0.67 & 14.60 & 0.67 & 40.42 & 0.40 & 23.10 & 0.58 \\
\hline Curvelet fuzzy & 17.49 & 0.61 & 13.87 & 0.64 & 41.90 & 0.43 & 24.42 & 0.56 \\
\hline
\end{tabular}

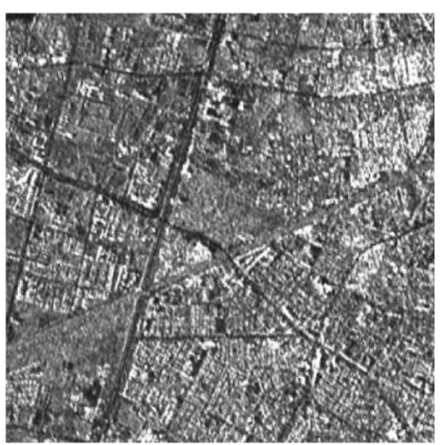

(a)

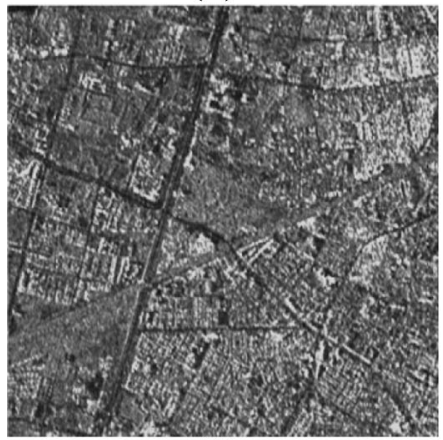

(d)

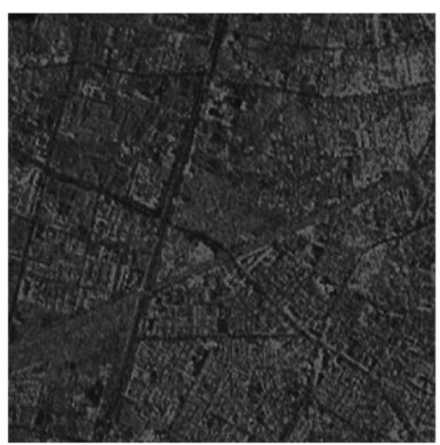

(b)

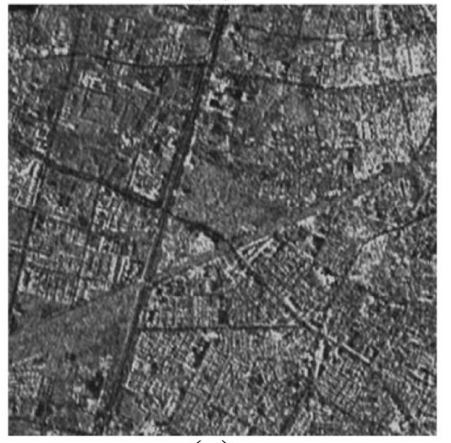

(e)

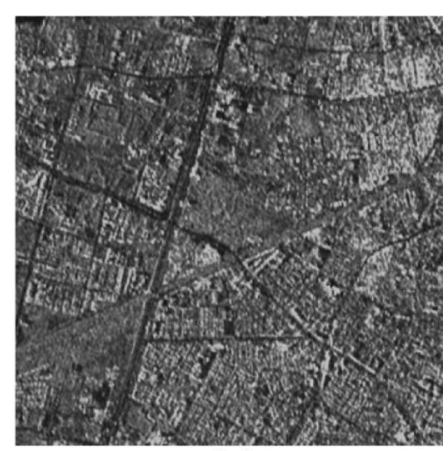

(c)

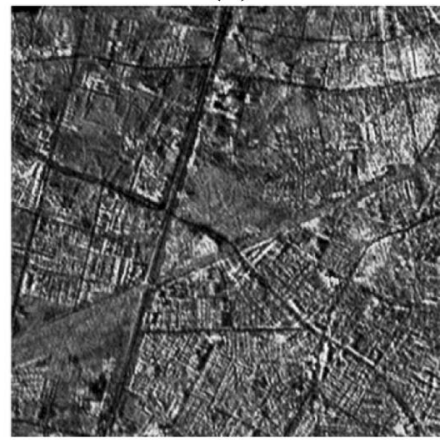

(f)

Figure 4. Denoising results of fine-resolution SAR image. (a) Original image, (b) wavelet -fuzzy, (c) curvelet denoising, (d) curvelet cycle spin, (e) curvelet improved soft and (f) curvelet fuzzy.

the ENL and SSI of these regions are measured. If the value of ENL is high the performance will be better, and if SSI is less then it suppresses speckle noise to a greater extent. The assessment parameter is measured over three uniform regions of a medium-resolution image as marked in figure 3 and are tabulated in table 2 .

The assessment parameter ENL and SSI is measured over three uniform regions tabulated in table 2. In region I, the ENL has 17.49, and it is comparatively higher than other techniques. As a result, it smoothens the region by removing more speckles compared to other methods. The SSI of the region I has 0.61 , which is less compared to other approaches. It results in reducing more amount of speckle in this region. In region II the ENL is 13.87, and the SSI is 0.43 in the region III, which results noise suppressed being less compared to curevelet improved soft technique; but compared to other methods, it removed more quantity of noise. But the average value of ESI and SSI is more compared with different methods. The average values of ENL and SSI of the proposed technique are 24.42 and 0.56, respectively, these values are better than those of other methods. As a result, its efficiency is more in smoothing the uniform region and also in suppressing more quantity of speckle noise.

By observing the visual quality of the image from figures 3 and 4 after denoising by different methods, the proposed method enhancing the uniform and non-uniform region also enhance the features present in the image by suppressing noise as well as improve the visual quality of the image compared to other methods. The results of the curvelet denoising technique and curvelet cyclespin are not competent because they use a hard threshold technique and 
induce some unwanted fuzzy edges, causing visual distortions in the homogeneous areas of the image.

\section{Conclusion}

In this work, the curvelet-based fuzzy technique is attempted to remove speckle noise. The fine- and mediumresolution images have better values of NMV, and NSD for the proposed approach results in less amount of speckle present in the denoised image. The MSD and ENL are taking higher values. It reflects in greater reduction in speckle from the non-uniform and uniform regions of the image. Moreover, the performance is outstanding. The metric SSI is having competent value, that is, less value, resulting in suppressing more speckle noise as observed with other approaches. Even in a uniform region, the ENL and SSI values are better than the other methods. As a result, the proposed method is quantitatively performing better. In addition, the quality of the image is improved by enhancing the features present in the image.

\section{Acknowledgement}

The authors would like to thank NRSC Hyderabad for providing free sample data set of RISAT-1.

\section{References}

[1] Qiu F, Berglund J, Jensen J R, Thakkar P and Ren D 2004 Speckle noise reduction in sar imagery using a local adaptive median filter. GISci. Remote Sens. 41(3): 244-266

[2] Goodman J W 1976 Some fundamental properties of speckle. J. Opt. Soc. Am. 66(11): 1145-1150

[3] Gonzalez R C and Woods R E 2008 Digital image processing. 3rd edn, Pearson Education, Upper Saddle River, London

[4] Lee J S 1980 Digital image enhancement and noise filtering by use of local statistics. IEEE Trans. Pattern Anal. Mach. Intell. 2(2): 165-168

[5] Frost V S, Stiles J A, Shanmugan K S and Holtzman J C 1982 A model for radar images and its application to adaptive digital filtering of multiplicative noise. IEEE Trans. Pattern Anal. Mach. Intell. 4(2): 157-166

[6] Kuan D T, Sawchuk A A, Strand T C and Chavel P 1985 Adaptive noise smoothing filter for images with signal-dependent noise. IEEE Trans. Pattern Anal. Mach. Intell. 7(2): 165-177

[7] Baraldi A and Parmiggiani F 1995 A refined gamma MAP SAR speckle filter with improved geometrical adaptivity. IEEE Trans. Geosci. Remote Sens. 33(5): 1245-1257

[8] Joshi R and Garg R D 2012 Pre-processing of TerraSAR-X data for speckle removal: an approach for performance evaluation. J. Indian Soc. Remote Sens. 40(3): 371-377

[9] Dellepiane S G and Angiati E 2014 Quality assessment of despeckled SAR images. IEEE J. Select. Topics Appl. Earth Observ. Remote Sens. 7(2): 691-707
[10] Donoho D L and Johnstone I M 1995 Adapting to unknown smoothness via wavelet shrinkage. J. Am. Stat. Assoc. 90(432): 1200-1224

[11] Donoho D L 1995 De-noising by soft-thresholding. IEEE Trans. Inform. Theory 41(3): 613-627

[12] Huimin C, Ruimei Z and Yanli H 2012 Improved threshold denoising method based on wavelet transform. International Conference on Medical Physics and Biomedical Engineering

[13] Xie H, Pierce L E and Ulaby F T 2002 SAR speckle reduction using wavelet denoising and markov random field modeling. IEEE Trans. Geosci. Remote Sens. 40(10): 2196-2212

[14] Wu Y, W Xia and Liao G-S 2006 SAR images despeckling based on Bayesian estimation and fuzzy shrinkage in wavelet domains. Chin. J. Aeronaut. 19(4): 326-333

[15] Amirmazlaghani M and Amindavar H 2009 A novel wavelet domain statistical approach for denoising SAR images. In: Proceedings of the International Conference on Image Processing, November, pp. 3861-3864

[16] Solbo S and Eltoft T 2008 A stationary wavelet-domain wiener filter for correlated speckle. IEEE Trans. Geosci. Remote Sens. 46(4): 1219-1230

[17] Candes E, Demanet L, Donoho D and Ying L 2006 Fast discrete curvelet transforms. Multiscale Model. Simul. 5(3): 861-899

[18] Liu Y, Gui Z and Zhang Q 2013 Noise reduction for lowdose X-ray CT based on fuzzy logical in stationary wavelet domain. Optik 124: 3348-3352

[19] Starck J, Candès E J and Donoho D L 2002 The curvelet transform for image denoising. IEEE Trans. Image Process. 11(6): 670-684

[20] Binh N T and Khare A 2010 Multilevel threshold based image denoising in curvelet domain. J. Comput. Sci. Technol. 25(3): 632-640

[21] Swamy S and Vani K 2015 SAR image enhancement using improved soft threshold function in curvelet domain. Int. J. Appl. Eng. Res. 10(9): 6756-6758

[22] Guo Y and Bai Z 2008 A new denoising method of SAR images in curvelet domain. In: 10th International Conference on Control, Automation, Robotics and Vision Hanoi, Vietnam, pp. 17-20

[23] Chen Z, Wang S, Fang G and Wang J 2013 Ionograms denoising via curvelet transform. Adv. Space Res. 52: 1289-1296

[24] Jin J, Yuan J, Shen Q, Yu Y, Zhou Y and Wang Y 2014 Curvelet transform based adaptive image deblocking method. Comput. Electr. Eng. 40(8): 117-129

[25] Su J, Wang B, Hsu T, Chou C and Tseng V S 2010 Multimodal image retrieval by integrating web image annotation, concept matching and fuzzy ranking techniques. Int. J. Fuzzy Syst. 12(2): 136-149

[26] Glaister J, Wong A and Clausi D A 2014 Despeckling of synthetic aperture radar images using Monte Carlo texture likelihood sampling. IEEE Trans. Geosci. Remote Sens. 52(2): 1238-1248

[27] Nasri M and Nezamabadi-pour H 2009 Image denoising in the wavelet domain using a new adaptive thresholding function. Neurocomputing 72: 1012-1025 